\title{
Component importance assessment of power systems for improving resilience under wind storms
}

\author{
Gengfeng LI ${ }^{1}$, Gechao HUANG ${ }^{1}$, Zhaohong BIE $^{1}$ (D), Yanling LIN ${ }^{1}$, \\ Yuxiong HUANG ${ }^{1}$
}

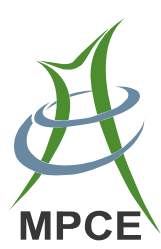

\begin{abstract}
Increasingly frequent natural disasters and manmade malicious attacks threaten the power systems. Improving the resilience has become an inevitable requirement for the development of power systems. The importance assessment of components is of significance for resilience improvement, since it plays a crucial role in strengthening grid structure, designing restoration strategy, and improving resource allocation efficiency for disaster prevention and mitigation. This paper proposes a component importance assessment approach of power systems for improving resilience under wind storms. Firstly, the component failure rate model under wind storms is established. According to the model, system states under wind storms can be sampled by the non-sequential Monte Carlo simulation method. For each system state, an optimal restoration model is then figured out by solving a component repair sequence optimization model considering crew
\end{abstract}

CrossCheck date: 14 May 2019

Received: 16 November 2018/Accepted: 14 May 2019/Published online: 9 July 2019

(C) The Author(s) 2019

$\triangle$ Zhaohong BIE

zhbie@mail.xjtu.edu.cn

Gengfeng LI

gengfengli@xjtu.edu.cn

Gechao HUANG

hgc096710@stu.xjtu.edu.cn

Yanling LIN

linyanling@stu.xjtu.edu.cn

Yuxiong HUANG

hyx.xj@stu.xjtu.edu.cn

1 The State Key Laboratory of Electrical Insulation and Power Equipment, The Shaanxi Key Laboratory of Smart Grid, Xi' an Jiaotong University, Xi' an 710049, China dispatching. The distribution functions of component repair moment can be obtained after a sufficient system state sampling. And Copeland ranking method is adopted to rank the component importance. Finally, the feasibility of the proposed approach is validated by extensive case studies.

Keywords Component importance, Resilience, Copeland score, Non-sequential Monte Carlo simulation

\section{Introduction}

Reliability is one of the basic requirements on the power supply. Recently, with the rapid development of theories, technologies, equipment, etc., the reliability performance of the power systems has been improved significantly [1]. However, when facing extreme events such as natural disasters and man-made attacks, power systems do not always perform well [2]. Lessons learned from some catastrophic accidents indicate that power outage sometimes is unavoidable [3], and thus the power systems need to be resilient, namely to be able to quickly restore from disasters [4].

Generally, extreme events have severe consequence but very low occurrence probability [5]. However, wind storm is a kind of natural disasters with relatively high frequency in coastal areas. For instance, the southeastern coastal areas of China are prone to wind storm disasters, e.g. in Guangdong Province, the average annual economic loss due to the power loss is immense because of the $4300 \mathrm{~km}$ coastline [6-8]. In most countries, the coastal areas tend to be the economic centers, and the effects of blackouts caused by wind storms can be more detrimental in these areas than in inland areas. Therefore, it is of great significance to investigate the efficient restoration strategy of 
power systems under wind storms to improve the resilience of the power systems.

When it comes to the resilience improvement strategy, there are two kinds of measures: hardening measures and operational measures [9]. These two are related to infrastructure and operational resilience respectively and both have positive effects on the resilience metrics [10]. The hardening measures which are efficient but costly include undergrounding distribution and transmission lines, upgrading poles and structures with stronger materials, etc. Reference [11] puts forward three kinds of resilience improvement strategies and analyzes which one should be adopted in different situations. Apparently, the result of the component importance assessment can provide us efficient and economic hardening strategies to improve the resilience of the power systems. The operational measures which are flexible and relatively cheap include advanced and accurate weather forecast, advanced and adaptive restoration, etc [11]. For example, extreme disasters such as wind storms usually cause multiple components fault at the same time [12]. Due to the limitation of repair crews and resources, those damaged components cannot be repaired at the same time. Obviously, the sequence of the repair will influence the restoration time so that the importance of the components should be evaluated. The component importance assessment can provide an efficient repair scheme.

There have been some preliminary research results about the problem. Firstly, probabilistic models such as the occurrence, intensity and moving speed of wind storms are established in [13], and then the component failure model under wind storms is introduced. Reference [14] puts forward a component failure model using quadratic approximation and exponential fitting. Those studies lay the foundation for the acquiring system states. When it comes to component importance assessment, most researches focus on the reliability fields which consider the typical outages. However, the component importance assessment based on resilience is seldom considered. When extreme events happen, qualitative repair strategies are often adopted in practice such as the nearest restoration strategy, the strategy based on the capacity, etc [15]. But the qualitative strategy cannot guarantee the best result. Aiming at the restoration after the disaster, [16] proposes a post-disaster restoration model based on a joint optimization of repair crew and DG dispatching. The proposed model is validated to be effective in distribution network. Reference [17] puts forward a service restoration model with mixedinteger second-order cone programming. However, these restoration optimization models may take a long time to solve, and should be defined as an offline analysis. Based on the offline optimization, the importance ranking of components can be obtained and used further to quickly figure out restoration strategies to improve the resilience of power system when facing a sudden disaster. Reference [18] proposes a component importance measure based on Copeland ranking method for critical infrastructure networks. However, the effects of specific disasters such as wind storms, and restoration optimization considering the repair crew dispatch are not included in the ranking process.

Therefore, a component importance assessment approach of power systems for improving resilience under wind storms is proposed in this paper. The approach can provide a component importance ranking method to improve resilience for power systems in advance, so that an optimal restoration strategy can be quickly acquired without long-time calculation when the disasters happen. Extensive case studies are conducted on IEEE standard test systems to validate the proposed approach, and several comparisons are carried out to illustrate the feasibility of the approach. The major contributions of this paper are as follows.

1) Aiming at improving the resilience, an optimal restoration model considering the repair crew dispatch based on the complex network theory is established. And the calculation results with different locations of the repair depot can give designers meaningful reference in the planning perspective.

2) The component importance ranking result can be obtained with the resilience-based assessment approach presented in this paper.

3) The model presented in this paper can provide a quantitative restoration strategy which has a compromise effect between resilience improvement and calculation efficiency.

This paper is organized as follows: Section 2 describes the component importance assessment of power systems based on resilience. The case studies are discussed in Section 3, and the conclusion is presented in Section 4.

\section{Component importance assessment of power systems based on resilience}

As mentioned above, the proposed component importance assessment approach of power systems for improving resilience under wind storms can be illustrated in Fig. 1.

The proposed approach includes two stages: ranking result can be obtained offline at pre-disaster stage, so that an optimal restoration strategy can be acquired easily and immediately at restoration stage. The main procedure of the proposed approach is presented as follows. 


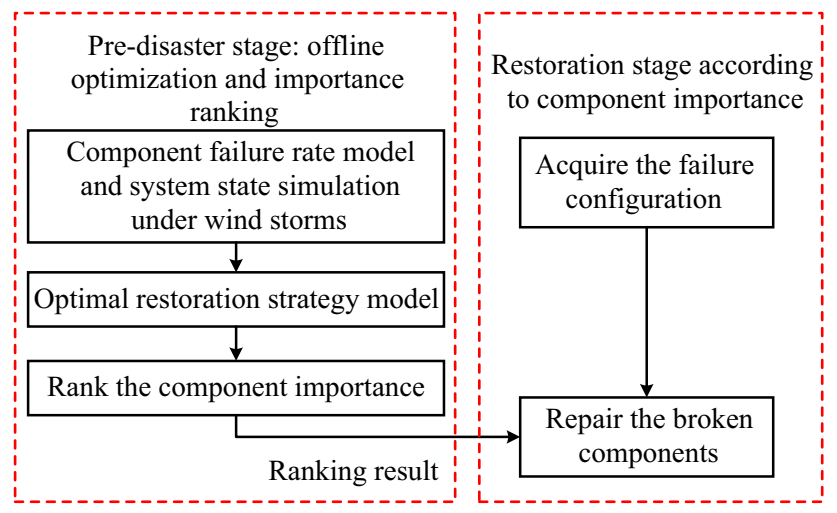

Fig. 1 Whole process of the assessment

\subsection{Power systems resilience}

The whole process of a wind storm affecting a power system is usually divided into 3 stages: pre-disaster stage, during-disaster stage, and post-disaster stage. A quantifiable and time-dependent system performance function $F(t)$ is used to analyze the whole process [18]. As shown in Fig. 2, before time $t_{e}$ the system is in normal operation, and at time $t_{e}$ the system encounters the disaster so that the system performance begins to deteriorate. At time $t_{d}$, the system performance reaches its minimum. The restoration process starts at time $t_{s}$ and ends at time $t_{f}$, at which time the system reaches a new level $F\left(t_{f}\right) . F\left(t_{f}\right)$ can be the same, close to or better than the original system performance $F\left(t_{0}\right) . F_{n}(t)$ is a curve which denotes the system performance if there is no disaster. Due to the outside disturbance, it is supposed to be time-dependent. In order to simplify the problem, it is assumed to be constant and equal to the system performance before the disaster, i.e., $F_{n}(t) \equiv F\left(t_{0}\right)$.

$F\left(t_{0}\right)-F\left(t_{d}\right)$ denotes the maximum performance loss when the disaster happens. $F(t)-F\left(t_{d}\right)$ denotes the performance which has been recovered at time $t$. Their ratio represents the recovered percentage of the system performance at time $t$. We define $R(t)$ as the system resilience at

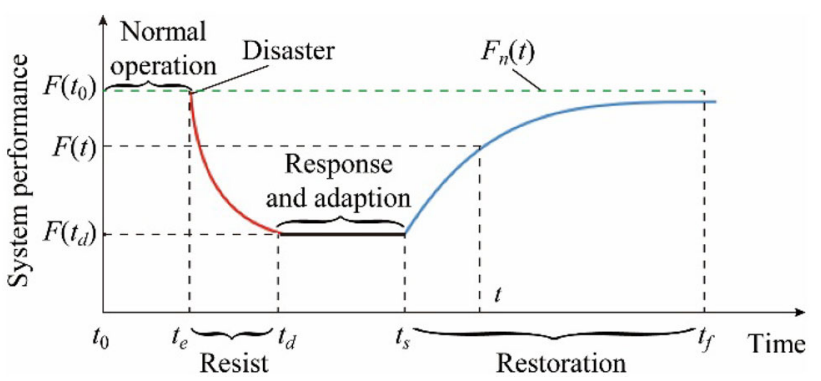

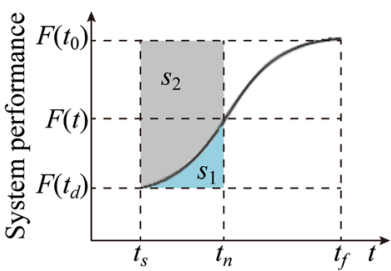

(a) Resilience at time $t_{n}$

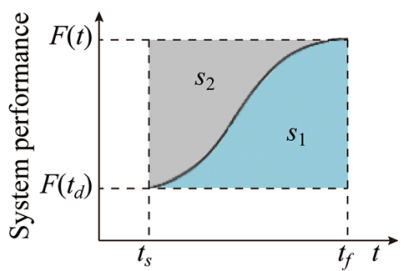

(b) Resilience of whole restoration process
Fig. 3 Resilience at different time

time $t . R(t)$ represents the cumulate percentage of the restored performance, which equals to the ratio of $S_{1}$ to $S_{1}+S_{2}$ as shown in Fig. 3 .

$R(t)=\frac{S_{1}}{S_{1}+S_{2}}=\int_{t_{s}}^{t} p(t) \mathrm{d} t=\int_{t_{s}}^{t} \frac{F(t)-F\left(t_{d}\right)}{F\left(t_{0}\right)-F\left(t_{d}\right)} \mathrm{d} t$

Natural disasters and man-made attacks usually impact on multiple components, and there will be different restoration schemes. This paper focuses on the effect of component restoration sequence on the restoration process. In order to guarantee the highest restoration efficiency and the best restoration result during the whole restoration process, the restoration scheme should maximize the resilience, that is, to make the area of $S_{1}$ as large as possible in the restoration process. This can be achieved by optimizing the restoration process, which will be introduced in Section 2.3.

\subsection{Component failure rate model and system state simulation under wind storms}

In order to accurately simulate the restoration process, we need to model the impact of wind storms on the system state by cooperating the storm speed with the failure rate [19].

\subsubsection{Component failure rate model under wind storms}

During a wind storm, as the pressure on trees increases, trees are more likely to fall on the overhead lines and damage the lines. Besides, the friction between the tower and wind, the lines and wind will increase, which will directly cause the tower and the lines to fall down or to contact other objects. Therefore, the wind could have large effect on the transmission line failure rate. The exponential fitting method to model the component failure rates under the wind storms is adopted [14]:

$\lambda_{\text {wind }}(w(t))=\left(\gamma_{1} \mathrm{e}^{\gamma_{2} w(t)}-\gamma_{3}\right) \lambda_{\text {norm }}$

where $w(t)$ is the wind speed at time $t ; \lambda_{\text {wind }}(w(t))$ is the failure rate of components under the wind speed of $w(t)$;

Fig. 2 System performance during the disaster 
$\lambda_{\text {norm }}$ is the failure rate of components in normal condition; and $\gamma_{1}, \gamma_{2}, \gamma_{3}$ are the fitting coefficient.

After the extreme wind storms, we can obtain the failure information via supervisory control and data acquisition (SCADA) and climate data via the weather station. With those data, the curve representing the relationship between failure rate and wind speed can be drawn. The parameters in (2) can be obtained by fitting the curve. Figure 4 shows the relation between the component failure rate and the wind speed [14]. When the wind speed is under a specific wind speed, the component failure rate under wind storms almost equals to the component failure rate in normal condition, whereas when the wind speed is over that specific wind speed, the effect of the wind speed on the component failure rate cannot be ignored. Define that specific wind speed as critical wind speed and assume that, when the wind speed is under the critical wind speed, the component failure rate is immune. According to Fig. 4 and [14], the critical wind speed $w_{\text {crit }}=8 \mathrm{~m} / \mathrm{s}, \gamma_{1}=0.21$, $\gamma_{2}=0.49, \gamma_{3}=9.83$. With (2) and the parameters, the failure rate under certain wind speed can be calculated.

The relation between the failure rate and failure probability [20] is:

$p_{i j}=1-\mathrm{e}^{\lambda_{i j} T_{y}}$

where $p_{i j}$ is the failure probability of component $(i, j), \lambda_{i j}$ is the failure rate of component $(i, j)$; and $T_{y}$ is the time related to the failure rates.

\subsubsection{System state simulation based on Monte Carlo simulation}

Monte Carlo simulation is a mathematical experiment method based on probability and stochastic theory [21]. It is widely used in the reliability and resilience research of

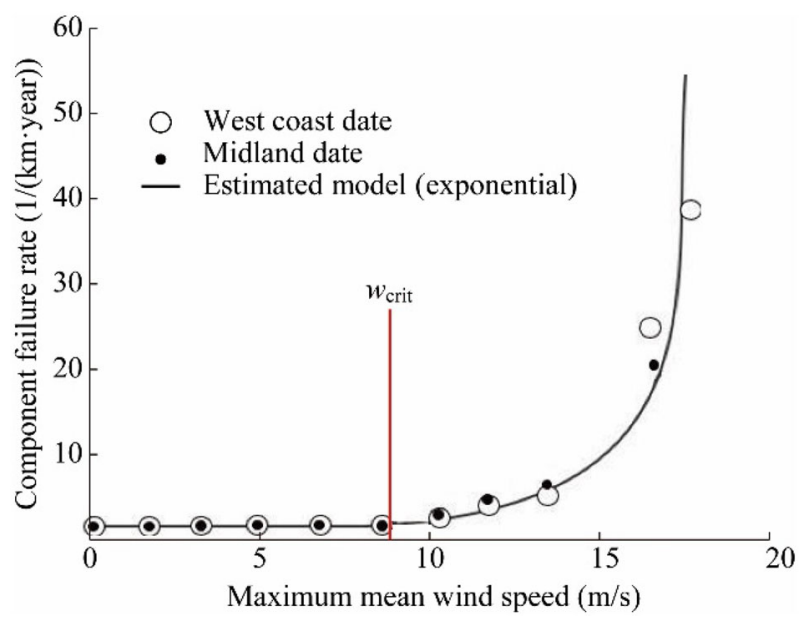

Fig. 4 Failure rate as a function of maximum mean wind speed (maximum value among mean wind speed of different periods) the power systems, and the detail information can be found in [10, 22-24]. In this paper, non-sequential Monte Carlo simulation method is adopted to sample power system state under wind storms.

Binary-variable $s_{i}(t)$ is defined as the state of component $i$ at time $t, s_{i}(t)=1$ if component $i$ works well at time $t$, otherwise $s_{i}(t)=0$. Firstly, for each sample, the state of each component $i$ at the beginning of restoration process (for simplicity, the start time of the restoration process is set to 0 ) can be determined by:

$s_{i}(0)= \begin{cases}1 & \text { if } \operatorname{rand}_{i}>p_{i} \\ 0 & \text { if } \operatorname{rand}_{i} \leq p_{i}\end{cases}$

where $s_{i}(0)=1$ means component $i$ works well at the beginning of restoration process, whereas $s_{i}(0)=0$ means component $i$ is damaged at the beginning of restoration process; rand $_{i}$ is a random number obeying uniform distribution generated for each sampling of component $i ; p_{i}$ is the failure probability of component $i$ and it can be derived according to the failure rate in (2) under a certain wind speed. Thus, $\boldsymbol{S}(0)=\left(s_{1}(0), s_{2}(0), \ldots, s_{n}(0)\right)$ which denotes a state of a power system with $n$ components at the beginning of the restoration process can be determined.

\subsection{Optimal restoration strategy model based on maximum resilience}

In this section, optimal restoration strategy model based on complex network theory will be introduced [25].

Complex network theory is an effective tool to study topological and kinetic properties of network [25]. Power systems and the net of the complex network theory have many things in common: (1) the buses and connecting components of a power system correspond to the vertex and the arcs of the network, respectively; (2) the power flows correspond to the flow in the network, and the transmission limitation of each component in power systems corresponds to the capacity limitation in the network. Therefore, in this paper, power systems are abstracted into a network in complex network theory.

In this paper, a network $G(V, E)$ is considered, where $V$ is the set of vertex and $E$ is the set of components. The vertex in the network are divided into three kinds: generator node $V_{S}$, transmission node $V_{T}$ and demand node $V_{D}$. $P_{i j}^{C}$ is the transmission capacity of the component $(i, j) \in E$, $P_{i}^{S}$ is the maximum power generated by the generator node $i \in V_{S}, P_{j}^{D}$ is the demand of demand node $j \in V_{D}$. The power is transmitted from the generator nodes to all the demand nodes and the power flow has to obey the physical constraints of the network. 


\subsubsection{Objective function}

When the disaster happens, the damaged components form a set $E^{\prime} \subseteq E$. In this paper, these components in $E^{\prime}$ are assumed to be damaged immediately after the disaster happens. The system performance reaches its minimum $F_{\min }$ at this time. The restoration process will start at $t=t_{s}$ (for simplification set $t_{s}=0$, so $F(0)=F_{\min }$ ). We define the sum of the power flows to the demand nodes as the system performance:

$F(t)=\sum_{j \in V_{D}} f_{j}(t)$

where $f_{j}(t)$ is the power flow received by the demand node $j$ at time $t$.

The objective of this model is to find out the repair sequence of fault components which can enable the system to obtain the maximum resilience during the restoration process. Assuming that the whole restoration process is a time-discrete process $(t=1,2, \ldots, T)$, and the value of time unit is determined by the practical demand. At each time unit, the repair crew can only repair a component or travel from one component to another. According to (1) and (5), we have:

$R(t)=\frac{\sum_{t=1}^{T}\left(\sum_{j \in V_{D}} f_{j}(t)-F_{\min }\right)}{T\left(\sum_{j \in V_{D}} P_{j}^{D}-F_{\min }\right)}$

where $\sum_{j \in V_{D}} P_{j}^{D}$ is the normal system performance.

In order to obtain the maximum resilience, the objective function is determined as:

$\max \frac{\sum_{t=1}^{T}\left(\sum_{j \in V_{D}} f_{j}(t)-F\left(t_{d}\right)\right)}{T\left(\sum_{j \in V_{D}} P_{j}^{D}-F\left(t_{d}\right)\right)}$

\subsubsection{Constraints}

In this model, constraints include component state, network capacity and crew travel distance.

\section{1) Component state constraint}

A two-state component model is adopted in this paper: $s_{i j}(t) \in[0,1], t=1,2, \ldots, T$. It shows the state of the component $(i, j) \in E$ at time $t$. The constraint conditions related to the state in [17] are:

$s_{i j}(t) \in\{0,1\} \quad \forall(i, j) \in E$ $s_{i j}(t) \leq s_{i j}(t+1) \quad \forall(i, j) \in E$

$s_{i j}(0)=0 \quad \forall(i, j) \in E^{\prime}$

Equation (8) shows that $s_{i j}(t)$ is a binary variable; (9) shows that the component will remain in operation once it is repaired; (10) shows that every component in $E^{\prime}$ is damaged at the beginning of the restoration process.

2) Network capacity constraint

The constraints related to the capacities of nodes and components are:

$\sum_{j \in V_{i}} f_{i j}(t)-\sum_{j \in V_{i}} f_{j i}(t) \leq P_{i}^{S} \quad \forall i \in V_{S}$

$\sum_{j \in V_{i}} f_{i j}(t)-\sum_{j \in V_{i}} f_{j i}(t)=0 \quad \forall i \in V_{T}$

$\sum_{j \in V_{i}} f_{i j}(t)-\sum_{j \in V_{i}} f_{j i}(t)=-f_{j}(t) \quad \forall i \in V_{D}$

$0 \leq f_{j}(t) \leq P_{j}^{D} \quad \forall i \in V_{D}$

$0 \leq f_{i j}(t) \leq s_{i j}(t) P_{i j}^{C} \quad \forall(i, j) \in E$

where continuous variable $f_{j}(t) \in R^{+}$is the power flow received by demand node $j$ at time $t$; continuous variable $f_{i j}(t) \in R^{+}$is the power flow transmitted from node $i$ to $j$ at time $t$; and $V_{i}$ denotes the node connected to the node $i$.

Equations (11)-(13) are the typical constraints of generator nodes, transmission nodes, and demand nodes: (11) shows the power generated by the generator nodes cannot exceed the maximum capacities; (12) and (13) are the energy balance constraints; (14) shows that the real supplied power to the demand node cannot exceed its demand; (15) limits the power flow transmitted through a component.

3) Crew travel distance constraint

After the disaster, the repair crew from depots will come to repair the broken components [16]. To make the component importance assessment result more convincing, we take the repair crew travel distance into consideration. The resilience in this paper is related to the whole restoration process so that we can not only consider the contribution of the component. If we first repair the component which has large contributions to the system but is far away from the depot, we may obtain less resilience in the whole process. Therefore, the time that it takes the repair crew to travel between two broken components in the restoration process is considered. Assume that the repair crew start from a depot. The constraints related to the distance and travel time are: 
$\sum_{n \in E^{\prime}, m \in E^{\prime}} x_{m, n}=1$

$\sum_{n \in E^{\prime}, m \in E^{\prime}} x_{n, m}=1$

$t_{m}^{a r r}+t_{m}^{r e}+t_{m, n}^{t r}-t_{n}^{a r r}=\left(1-x_{m, n}\right) M$

$\forall m \in E^{\prime} \cup\{d e p\}, \forall n \in E^{\prime}$

$t_{d e p}^{a r r}=0$

$\sum_{t=1}^{T} f_{m, t}=1 \quad \forall m \in E^{\prime}$

$\sum_{t=1}^{T} t f_{m, t}=t_{m}^{a r r}+t_{m}^{r e} \quad \forall m \in E^{\prime}$

$s_{m}(t)=\sum_{\tau=1}^{t} f_{m, \tau} \quad \forall \in E^{\prime}$

where the binary variable $x_{m, n} \in[0,1]$ denotes whether the repair crew travel from component $m$ to component $n$. If the repair crew travel from $m$ to $n, x_{m, n}=1$ or $0 ; M$ is a large number; dep denotes the depot; $t_{\text {dep }}^{a r r}$ denotes the moment when the repair crew arrive at depot; discrete variable $t_{m}^{a r r} \in[1,2, \ldots, T]$ denotes the moment the repair crew arrive at the component $m$; binary variable $s_{m}(t)$ denotes the state of component $m$ at time $t$; binary variable $f_{m, t} \in[0,1]$ denotes whether component $m$ is repaired or not at time $t ; t_{m}^{r e}$ represents how long it takes when the repair crew repair the component; and $t_{m, n}^{t r}$ denotes how long it takes when the repair crew travel from $m$ to $n$.

Equations (16) and (17) show that the repair crew can only arrive at one component once and leave from it once. Equation (18) makes sure the route for the repair crew is successive. The repair crew will spend $t_{m}^{r e}$ repairing the component after they arrive at $m$, and then they will also spend $t_{m, n}^{t r}$ arriving at $n$. Big-M method guarantees that the route is successive because if the route is not successive, there must be at least $x_{m, n}=0$ which will lead to huge $t_{n}^{a r r}$. When $t_{n}^{a r r}$ is huge, the solution cannot be the optimal solution. Equation (19) shows that repair crew leave from depot at the beginning of the process. Equation (20) shows that every broken component can only be repaired once. Equation (21) establishes the relation between $f_{m, t}$ and $t_{m}^{a r r}$. Equation (22) shows that the component works well after it is repaired.

The moment when the broken component $m$ is repaired is denoted as $T_{m}$ and it can be calculated by:

$T_{m}=t_{m}^{a r r}+t_{m}^{r e}=\sum_{t=0}^{T}\left(1-s_{i j}(t)\right) \quad m \in E^{\prime}$

The model presented above is a mixed integer linear programming (MILP) problem, and the Gurobi optimization solver is employed in this paper to solve it.

\subsection{Copeland ranking}

The cumulative distribution function (CDF) of the repair moment of each component can be obtained by solving the MILP problem for each sampled failure configuration. In order to rank the importance of the components, the Copeland ranking method is introduced. Copeland ranking is a non-parametric Condorcet method which is usually used in politics field. This method does not require much information about the data, and operates on a candidate pool in which every object has $\Omega$ characteristics. By doing pair-wise comparisons between objects in different $\Omega$ characteristics in the candidate pool, the scores of all the candidates can be calculated, and the candidates can be ranked based on this score. A modified Copeland method which can be used to rank the CDFs presented in [26] is adopted in this paper. Define the percentiles of the CDF as the $\Omega$ characteristics, so the Copeland scores $\left(S_{m}\right)$ of component $m$ can be obtained:

$S_{m, n, k}= \begin{cases}S_{m, n, k-1}+1 & \text { if } q_{k}(m) \succ q_{k}(n) \\ S_{m, n, k-1}+0.5 & \text { if } q_{k}(m)=q_{k}(n) \\ S_{m, n, k-1} & \text { if } q_{k}(m) \prec q_{k}(n)\end{cases}$

$S_{m}=\sum_{n \in E^{\prime}, m \in E^{\prime}} S_{m, n, \Omega}$

where $q_{k}(m)$ is the $k^{\text {th }}$ percentile of the CDF of the repair moment of component $m ; S_{m, n, k}$ is the Copeland score after the $k^{\text {th }}$ comparison between $m$ and $n$, $\forall m, n \in E^{\prime}, k=1,2, \ldots, \Omega$; and $S_{m}$ is the Copeland score of the component $m$. The symbol " $\succ$ " represents "better than" and in this case, "better than" means "earlier than"; and the symbol " $\prec$ " represents "later than" in this case.

\subsection{Detailed procedures of the approach}

To sum up, the detailed procedure of the component importance assessment approach is shown in Fig. 5.

1) Initialize the parameters of network $G(V, E)$ : the demand of the demand nodes $P_{j}^{D}$, the maximum generating power of the generator nodes $P_{i}^{S}$, the transmission capacity of the components $P_{i j}^{C}$, the repair time of the component $(i, j) \in E\left(t_{i j}^{r e}\right)$, the travel time $t r_{m, n}$ between $m$ and $n$, and the location of the depot.

2) Use Monte Carlo simulation method to sample the failure configuration. When disaster scenarios are considered, too many damaged components will make the ranking meaningless, whereas too few damaged components cannot give us a creditable ranking result. 


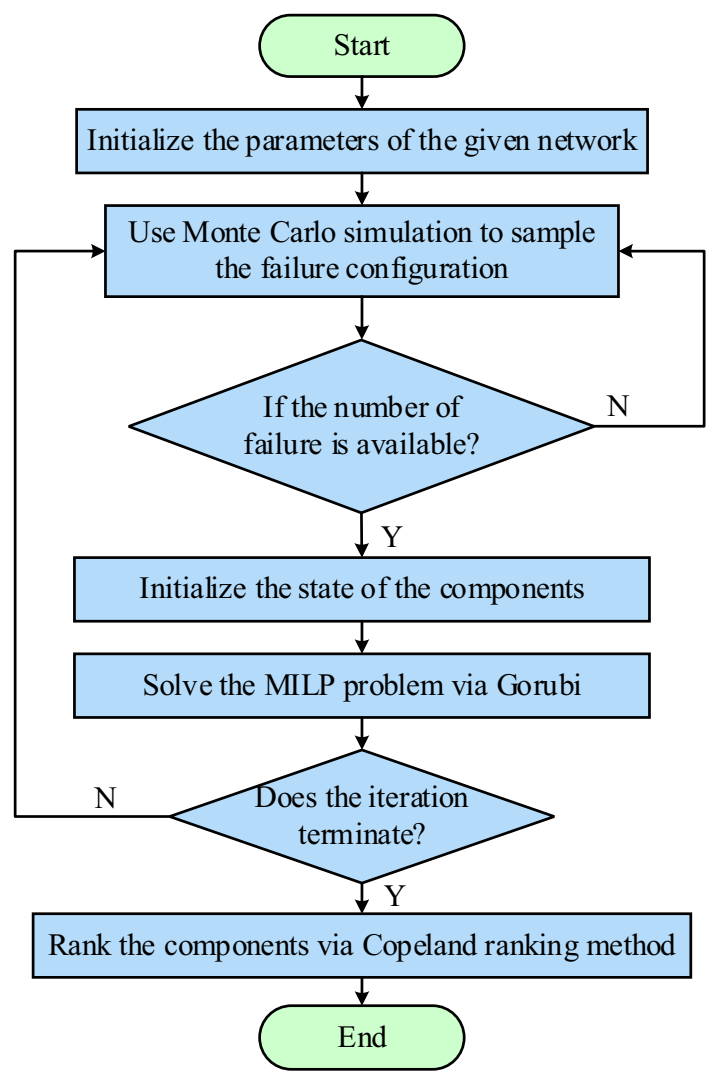

Fig. 5 Algorithm flow chart

Therefore, in this paper, we consider the scenario where 8-10 components fail at the same time. Once an appropriate disaster scenario is generated, set the states of the damaged components to zero.

3) Solve the MILP problem by Gurobi to obtain the best restoration sequence, and record the repair moment of each component.

4) Repeat the above process $N$ times, and obtain the CDF of the repair moment of each component.

5) Use Copeland ranking method to rank the importance of the component. According to the result of the Copeland ranking, the component importance ranking result is obtained.

\section{Case studies}

The proposed resilience-based component importance assessment approach is conducted on two test systems: IEEE 14-bus system and IEEE RTS 24-bus system.

\subsection{IEEE 14-bus system}

The IEEE 14-bus system, which contains 14 nodes and 20 lines, is converted into a topological network consisting

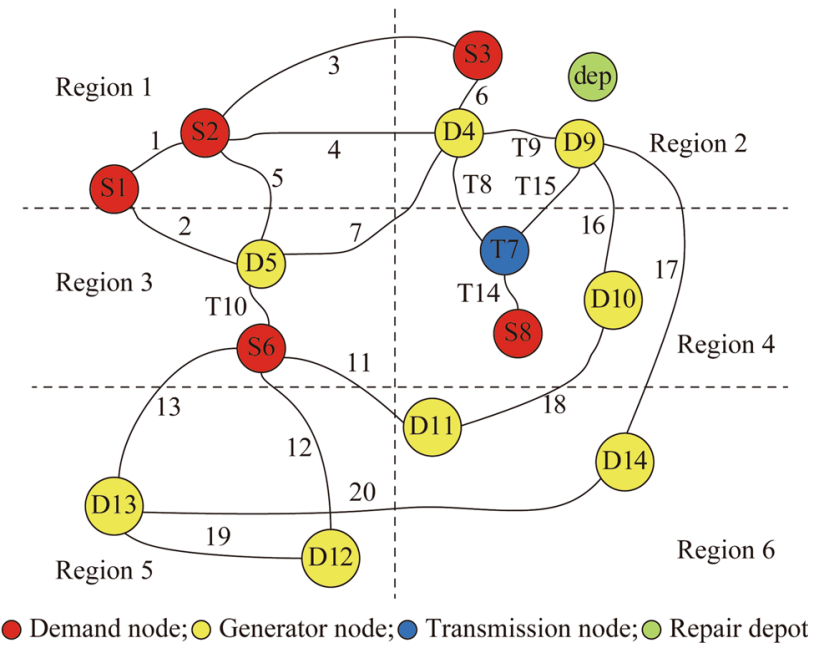

Fig. 6 Abstract topological network of IEEE 14-bus system

of nodes and edges, as shown in Fig. 6. Nodes are divided into three types: generator nodes, demand nodes, and transmission nodes. The strategy and time of the repair crew travel are affected by the distance between components. As shown in Fig. 6, the entire IEEE 14-bus network is divided into six regions. The distance between two adjacent areas is defined as one distance unit. Here, the travel time of the repair crew between two components is defined as follows:

1) Moving within the same region does not take time.

2) Moving between areas with one distance unit (e.g. region 1 and region 2) costs one time unit.

3) Moving between regions with two distance units (e.g. region 1 and region 5) or $\sqrt{2}$ distance units (e.g. region 1 and region 4) costs 2 time units.

4) Moving between regions with $\sqrt{5}$ distances units (e.g. region 1 and region 6) costs 3 time units.

Assuming the system is operating normally, its parameters are shown in Table 1 . The value of power unit is determined by the practical demand. The location of the depot is set at region 2. The failure rate data come from [14]. The resilience-based component importance assessment approach proposed in the Section 2 is applied in this system.

Table 1 Initial parameters for IEEE 14-bus system

\begin{tabular}{lll}
\hline Parameter & Symbol & Value (units) \\
\hline Capacity of generator node & $P_{i}^{S}$ & 16 \\
Demand of demand node & $P_{j}^{D}$ & 10 \\
Capacity of transmission node & $P_{i j}^{C}$ & 16 \\
\hline
\end{tabular}




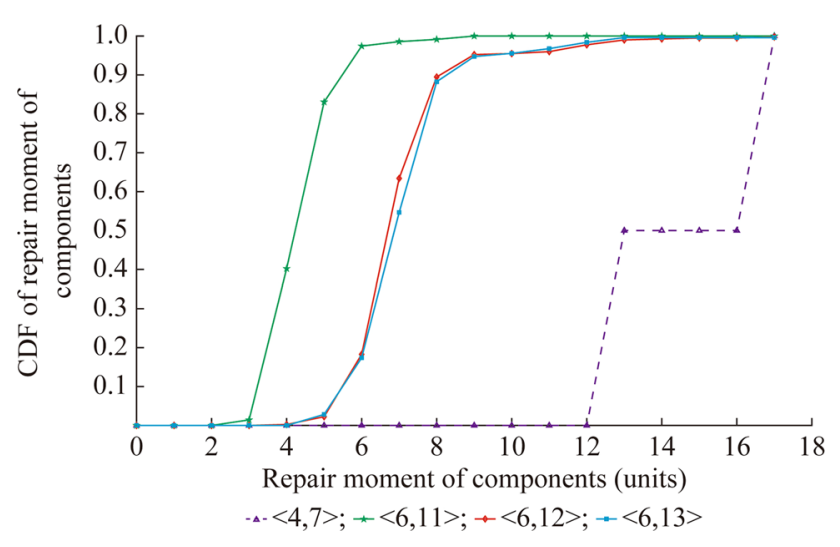

Fig. $7 \mathrm{CDF}$ of repair moment of five representative components

After a number of simulations (1000 times in this paper), CDF of the repair moment of 20 components can be obtained. Figure 7 shows the CDF of the repair moments of five representative components. It can be seen that the repair time of component $<6,11>$ (the line between node 6 and node 11 ) is always smaller than 8 , and the repair time of component $<4,7>$ will always be greater than 12 . Obviously, the component $<6,11>$ can be considered as more important than the component $<4,7>$, because with component $<6,11>$ being repaired earlier than component $<4,7>$, the system will obtain a larger resilience value.

However, not all the relative importance of the components can be judged so intuitively. For example, the importance relationship between the components $<6,12>$ and the components $<6,13>$ is difficult to judge since their distribution functions intersect. Therefore, Copland ranking method can be adopted to rank the importance of these kinds of components. Figure 8 shows the Copland score of each component in the IEEE 14-bus system.

It can be seen from Fig. 8 that element $<3,4>$ has the highest Copland score, while element $<4,7>$ has the lowest Copland score. There are two types of components with higher scores: (1) components connecting two areas e.g. $<4,9>,<5,6>,<7,9>$; (2) demand nodes and

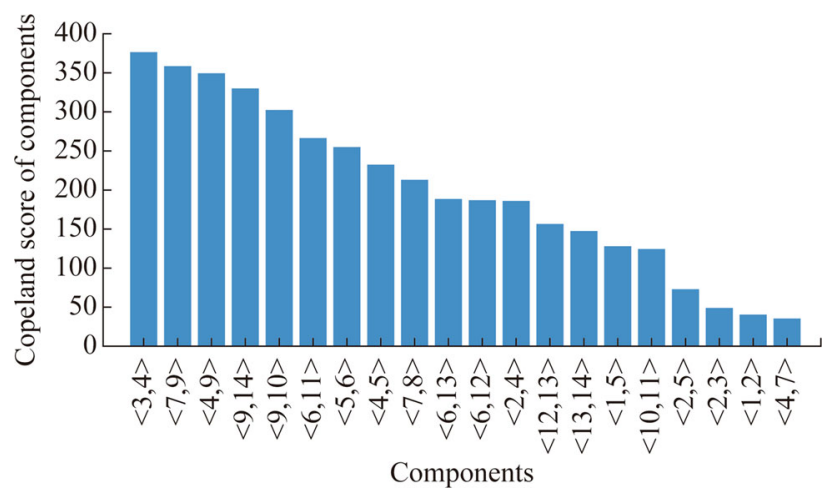

Fig. 8 Results of Copeland ranking method in IEEE 14-bus system generator nodes that are close to the depots e.g. $<3,4>,<6$, $11>$. There are two types of components that have lower scores: (1) components between generator nodes e.g. $<1$, $2>,<2,3>$; (2) there are multiple components between two nodes. Then these components have lower scores e.g. $<2$, $4>,<2,5>$. Components with high Copland score should have a higher priority in the restoration process, which can make the entire restoration process more efficient.

\subsection{IEEE RTS 24-bus system}

The IEEE RTS 24-bus system, which includes 24 nodes and 34 lines, is used as a test example at this section to illustrate the superiority of the proposed method. Similarly, the test system is converted into a topological network consisting of nodes and edges, as shown in Fig. 9. Assuming that the system is operating normally, its parameters are shown in Table 2. The location of the depot is set in region 4 .

\subsubsection{Effect of different locations of the depot}

To illustrate the effect of different locations of the depot on the component importance assessment result, we compare the component importance ranking results when depot is set in region 4 and region 2 .

This paper concentrates on the resilience improvement and component importance assessment in the operational perspective while the location of the crew depot is more

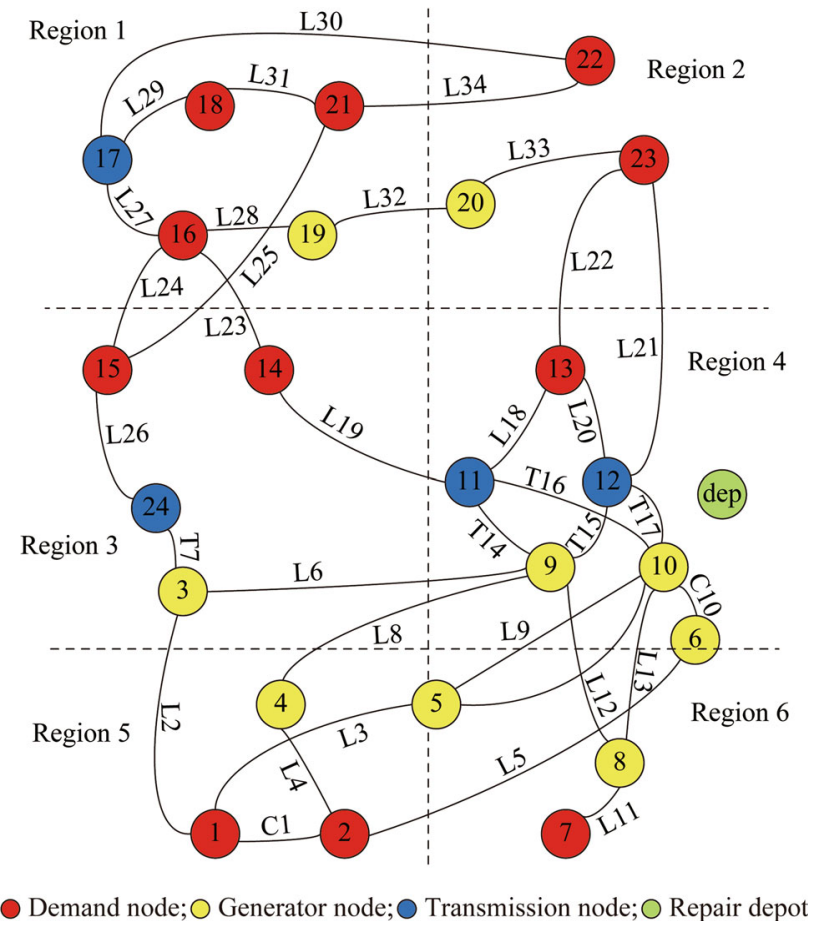

Fig. 9 Abstract topological network of IEEE RTS 24-bus system 
Table 2 Initial parameters for IEEE RTS 24-bus system

\begin{tabular}{lll}
\hline Node & Symbol & Value (units) \\
\hline Capacity of the generator node & $P_{i}^{S}$ & 9 \\
Demand of the demand node & $P_{j}^{D}$ & 11 \\
Capacity of the transmission node & $P_{i j}^{C}$ & 18 \\
\hline
\end{tabular}

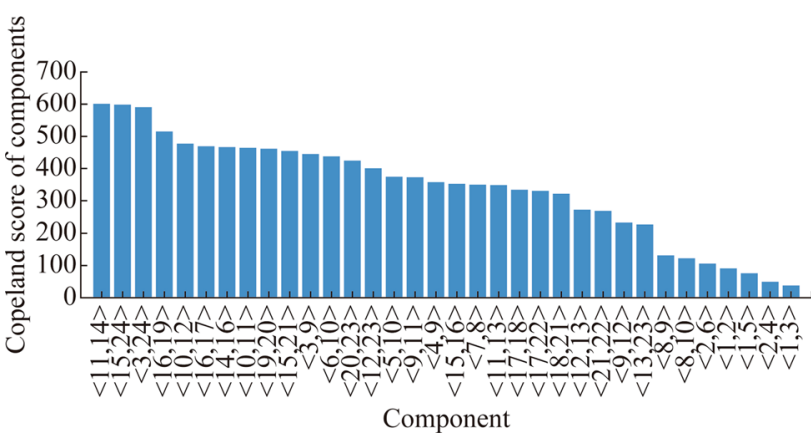

Fig. 10 Results of Copeland ranking method when depot is in region 4

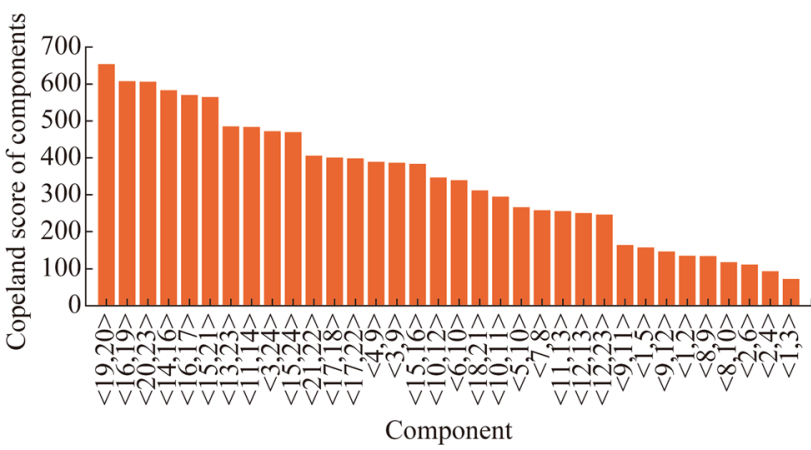

Fig. 11 Results of Copeland ranking method when depot is in region 2

related to the planning perspective. As shown in Figs. 10 and 11 , the most important component is $<11,14\rangle$ when the depot is in region 4 while it is $\langle 19,20\rangle$ when the depot is in region 2. Almost all components' importance change when the location of the depot change. Conclusions can still be drawn that: (1) different depot locations will have effect on the component importance ranking result; (2) with the approach proposed in this paper, a meaningful planning reference can be provided for the designers because different repair sequence will lead to different load shedding.

\subsubsection{Optimal restoration strategy, nearest restoration strategy and offline optimization strategy}

In the optimal restoration strategy, we can obtain the strategy which can make the resilience of the power systems maximal. But this strategy may take long time to solve, which may lead to a long-time blackout. In the offline optimization strategy, we use the strategy which is obtained with the offline component importance assessment. Although the resilience of the power systems may cannot reach its maximum, we can save much time and start the restoration process in time. In the nearest restoration strategy, the faulty component closest to the depot is repaired first, but this repair strategy does not consider the load shedding and the repair time in the entire restoration process. To illustrate the feasibility of offline optimization strategy, the load shedding and repair time for the three strategies are compared.

In this case study, the Monte Carlo simulation method is used to generate another set of fault scenarios where the three restoration strategies are adopted.

The CDFs of the load shedding and the time of repair process are shown in Fig. 12. The expected value of load shed and the expected time of repair time for the three strategies are shown in Table 3. From Table 3, we can see that using the optimal restoration strategy and offline optimization strategy for restoration can significantly reduce the load shedding and time of restoration process. Compared with the offline optimization strategy, optimal restoration strategy can slightly reduce the load shedding and the repair time but take much more time. So, using the offline optimization strategy can significantly reduce the load shedding and the repair time while taking much less time, which can be adopted in the practical restoration process.

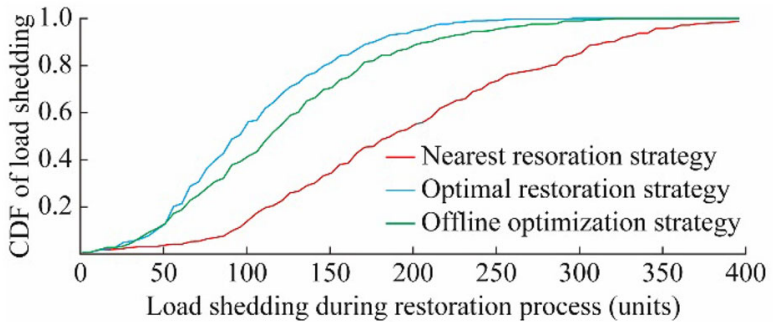

(a)

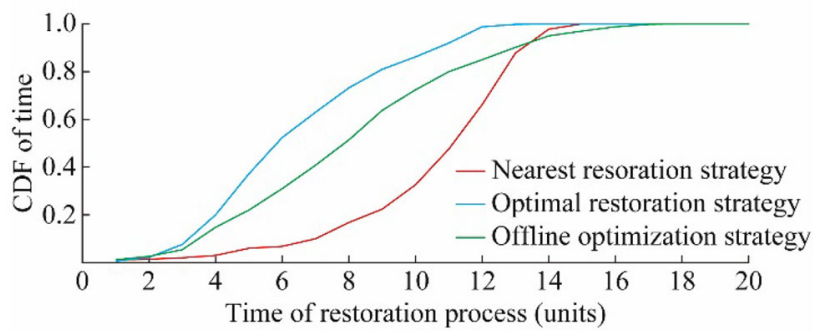

(b)

Fig. $12 \mathrm{CDF}$ of load shedding in whole restoration process and CDFs of restoration time for three strategies 
Table 3 Expected value of load shedding and restoration time

\begin{tabular}{llc}
\hline Strategy & $\begin{array}{l}\text { Expected value of load shedding } \\
\text { (units) }\end{array}$ & Expected value of restoration time (units) \\
\hline Nearest restoration strategy & 194.8940 & 10.9860 \\
Optimal restoration strategy & 102.9700 & 6.8600 \\
Offline optimization strategy & 120.6760 & 8.4880 \\
\hline
\end{tabular}

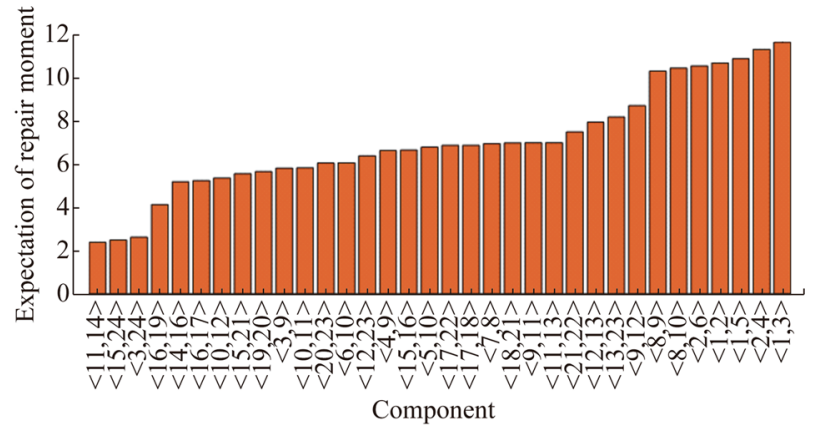

(a)

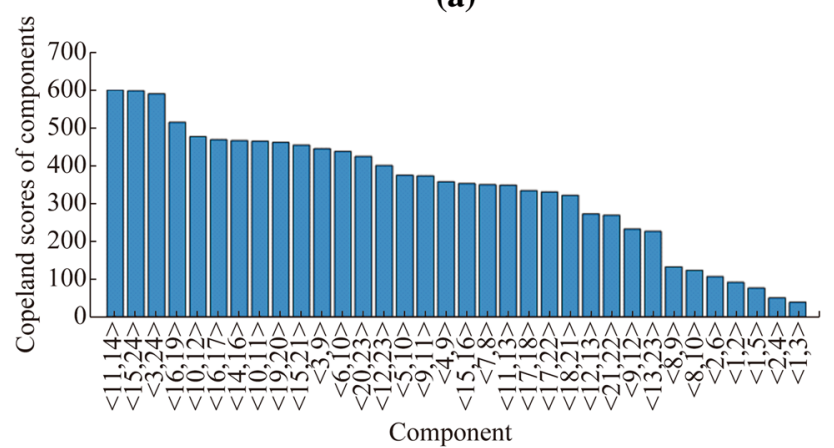

(b)

Fig. 13 Ranking results using different ranking methods

\subsubsection{Expectation ranking and Copland ranking}

Once the ranking of the components is obtained, if a disaster happens, the system operators can schedule the repair crew to the damaged components according to the ranking sequence. Those components with a higher ranking score will be repaired earlier. These strategies can be implemented in an online fashion.

In this section, we would like to test another ranking method. After obtaining the CDF of the restoration moment of each component, the expected moment of each component being repaired can be obtained, and then the components can be ranked according to the expected repair moments.

The differences between the two ranking methods are compared in this section. The importance ranking results of the two ranking methods are shown in Fig. 13. Then the Monte Carlo simulation method is used for sampling a new

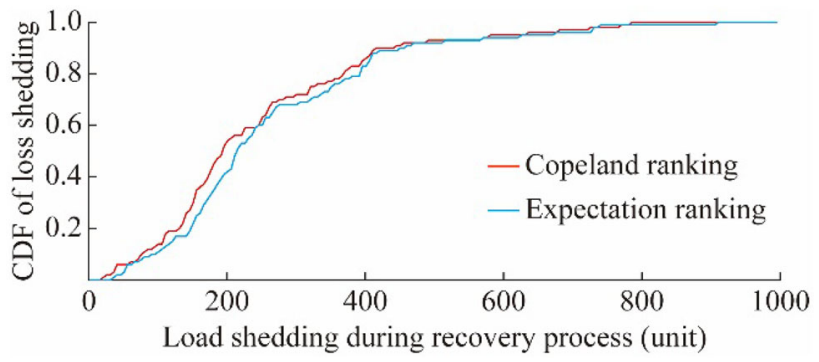

Fig. 14 CDFs of load shedding in the whole restoration time using different ranking methods

set of disasters, the system is restored according to the two ranking sequences respectively, and the load shedding and repair time are calculated. The CDFs of load shedding are shown in Fig. 14. The expected value of load shedding is 244.2700 units when the Copland ranking sequence is used. The expected value of load shedding is 263.9300 units when the expectation ranking sequence is used. The results show that the load shedding using Copeland ranking sequence is less than the load shedding using the expectation ranking method (not by a significant order). This indicates that both two methods can be adopted, but the Copeland ranking method promises better performance.

\section{Conclusion}

This paper puts forward a component importance assessment approach of power systems based on resilience under wind storms. Its advantage is that both the resilience and the repair crew dispatch are considered. At every simulation process, we solve a MILP and after a number of times simulation processes, the cumulative distribution function of repair moment of each component is obtained. Then the Copeland ranking method is used to rank the components.

Several conclusions can be drawn: (1) the proposed offline optimization strategy based on the component importance assessment has a compromise effect between resilience improvement and calculation efficiency; (2) the result of Copeland ranking method is slightly better than expectation ranking method; (3) the component importance 
assessment approach under wind storms can give a practical and effective restoration strategy reference and a meaningful planning reference. In the future, more actual factors such as the start-stop of generators and specific time dimension should be considered when assessing component importance.

Acknowledgements This work is supported by Science and Technology Project of State Grid Corporation of China (No. 5202011600UG)

Open Access This article is distributed under the terms of the Creative Commons Attribution 4.0 International License (http:// creativecommons.org/licenses/by/4.0/), which permits unrestricted use, distribution, and reproduction in any medium, provided you give appropriate credit to the original author(s) and the source, provide a link to the Creative Commons license, and indicate if changes were made.

\section{References}

[1] Zhang H, Yuan H, Li G et al (2018) Quantitative resilience assessment under a tri-stage framework for power systems. Energies 11(6): 1427

[2] Wang Y, Chen C, Wang J et al (2016) Research on resilience of power systems under natural disasters-a review. IEEE Trans Power Syst 31(2):1604-1613

[3] Cong H, Yang HE, Wang X et al (2018) Robust optimization for improving resilience of integrated energy systems with electricity and natural gas infrastructures. J Mod Power Syst Clean Energy 6(5):1066-1078

[4] Lin Y, Bie Z, Qiu A (2018) A review of key strategies in realizing power system resilience. Glob Energy Interconnect 1(1):70-78

[5] Bie Z, Lin Y, Li G et al (2017) Battling the extreme: a study on the power system resilience. Proc IEEE 105(7):1253-1266

[6] Liu SJ, Liu C, Li XY et al (2012) The influence of typhoon on power load characteristics in Guangdong Province. Yunnan Electr Power 40(1):91-93

[7] Yu L (2011) Research on typhoon disaster loss evaluation and management in Guangdong Province. Econ Res Guide 2011(23):63-65

[8] Zheng G, Zhong H (2015) Risk analysis of typhoon impacting the power grid of Guangdong Province. Electr Age 3:80-83

[9] Panteli M, Trakas DN, Mancarella P et al (2017) Power systems resilience assessment: hardening and smart operational enhancement strategies. Proc IEEE 105(7):1202-1213

[10] Panteli M, Mancarella P, Trakas D et al (2017) Metrics and quantification of operational and infrastructure resilience in power systems. IEEE Trans Power Syst 32(6):4732-4742

[11] Ma S, Chen B, Wang Z (2018) Resilience enhancement strategy for distribution systems under extreme weather events. IEEE Trans Smart Grid 9(2):1442-1451

[12] Yamada H, Miura T, Chikui S (2012) Controlling networks with an intelligent power system-toward disaster-resilient networks. In: Proceedings of international symposium on wireless personal multimedia communications, Taipei, China, 24-27 September 2012, $1 \mathrm{pp}$

[13] Li G, Zhang P, Luh PB et al (2014) Risk analysis for distribution systems in the Northeast U.S. under wind storms. IEEE Trans Power Syst 29(2):889-898
[14] Alvehag K, Soder L (2011) A reliability model for distribution systems incorporating seasonal variations in severe weather. IEEE Trans Power Deliv 26(2):910-919

[15] Chen C, Wang J, Ton D (2017) Modernizing distribution system restoration to achieve grid resiliency against extreme weather events: an integrated solution. Proc IEEE 105(7):1267-1288

[16] Arif A, Wang Z, Wang J et al (2017) Power distribution system outage management with co-optimization of repairs, reconfiguration, and DG dispatch. IEEE Trans Smart Grid 9(5):4109-4118

[17] Li Y, Xiao J, Chen C et al (2018) Service restoration model with mixed-integer second-order cone programming for distribution network with distributed generations. IEEE Trans Smart Grid. https://doi.org/10.1109/TSG.2018.2850358

[18] Fang YP, Pedroni N, Zio E (2016) Resilience-based component importance measures for critical infrastructure network systems. IEEE Trans Reliab 65(2):502-512

[19] Hu B, Li Y, Yang H et al (2017) Wind speed model based on kernel density estimation and its application in reliability assessment of generating systems. J Mod Power Syst Clean Energy 5(2):220-227

[20] Enrico Z, Roberta P (2010) Randomized flow model and centrality measure for electrical power transmission network analysis. Reliab Eng Syst Saf 95:379-385

[21] Bie Z, Wang X (1997) Application of monte carlo method in evaluating power system reliability. Autom Electr Power Syst 21(6):68-75

[22] Yushi T, Das AK, Payman A et al (2018) Distribution systems hardening against natural disasters. IEEE Trans Power Syst 33(6):6849-6860

[23] Xiao R, Xiang Y, Wang L et al (2018) Power system reliability evaluation incorporating dynamic thermal rating and network topology optimization. IEEE Trans Power Syst 33(6):6000-6012

[24] Amin G, Tohid S, Hassan AM et al (2018) Toward a consensus on the definition and taxonomy of power system resilience. IEEE Access 6:32035-32053

[25] Wang H, Shan Z, Ying G et al (2016) Evaluation method of node importance for power grid considering inflow and outflow power. J Mod Power Syst Clean Energy 5(5):1-8

[26] Al-Sharrah G (2010) Ranking using the Copeland score: a comparison with the Hasse diagram. J Chem Inf Model 50(5):785-791

Gengfeng $\mathrm{LI}$ received the $\mathrm{Ph} . \mathrm{D}$. degree in electrical engineering from Xi'an Jiaotong University, Xi' an, China, in 2014. He is currently an associate professor of School of Electrical Engineering at Xi'an Jiaotong University. His research interests include power system reliability evaluation, integration of renewable energy, and power system resilience.

Gechao HUANG received the B.S. degree in electrical engineering from Xi' an Jiaotong University, Xi' an, China, in 2018. He is currently pursuing his M.S. degree at Xi'an Jiaotong University. His research interests include power system resilience and integration of renewable energy.

Zhaohong BIE received the B.S. and M.S. degrees in electrical engineering from Shangdong University, Jinan, China, in 1992 and 1994, respectively, and the Ph.D. degree from Xi'an Jiaotong University, Xi'an, China, in 1998. Currently, she is a professor in the State Key Laboratory of Electrical Insulation and Power Equipment and the School of Electrical Engineering, Xi'an Jiaotong University. Her research interests include power system reliability 
evaluation, integration of renewable energy, smart grid, microgrids, and cyber-physical energy system.

Yanling LIN received her B.S. degree in electrical engineering from Shandong University, China, in 2013. She is now pursuing her Ph.D. degree in Xi'an Jiaotong University, Xi'an, China. Her research interests include power system resilience, microgrid, and renewable energy integration.
Yuxiong HUANG received the B.S. degree in electrical engineering from Xi' an Jiaotong University, Xi' an, China, in 2017. He is currently pursuing his M.S. degree at Xi'an Jiaotong University. His research interests include integrated energy system reliability and power system resilience. 